\title{
Effects of diltiazem on platelet activation and cytosolic calcium during percutaneous transluminal coronary angioplasty
}

\author{
H Dai, J Chen, Q Tao, J Zhu, F Zhang, L Zheng, Y Qiu
}

Postgrad Med J 2003;79:522-526

See end of article for authors' affiliations

Correspondence to: Dr Jianhua Zhu Department of Cardiology, First Affiliated Hospital of Zhejiang University, No 79 Qingchun Road, Hangzhou, Zhejiang 310003, China zjh_john@sina.com

Submitted

19 January 2003

Accepted 6 April 2003

\begin{abstract}
Aims: To evaluate effects of diltiazem on platelet hyper-reactivity in situations associated with endothelial injury and their possible relationship to cytosolic calcium concentration.

Methods: Blood samples were collected at seven time points from 35 patients undergoing percutaneous transluminal coronary angioplasty (PTCA) who received combined diltiazem and aspirin/ ticlopidine therapy or aspirin/ticlopidine therapy alone. Platelet expression of glycoprotein $\mathrm{llb} / \mathrm{llla}$ and $P$-selectin, production of thromboxane $B_{2}$, and cytosolic calcium concentration were measured, respectively, by whole blood flow cytometry, radioimmunoassay, and fluorospectrophotometry. The effects of diltiazem of different concentrations on expression of glycoprotein IIb/IIla and P-selectin were also studied in vitro in blood samples from patients with chronic stable angina.

Results: Of the two treatments, aspirin/ticlopidine therapy did not prevent an acute increase of expression of glycoprotein $\mathrm{Ilb} / \mathrm{Illa}$ and P-selectin and plasma thromboxane $\mathrm{B}_{2}$ five minutes and 10 minutes after first inflation and 10 minutes after PTCA, whereas combined diltiazem and aspirin/ticlopidine therapy had a significant inhibitory effect. In the group receiving aspirin/ticlopidine therapy, there was a short term increase of platelet $\left[\mathrm{Ca}^{2+}\right]$ immediately after PTCA which was significantly reduced by diltiazem treatment. Expression of glycoprotein $\mathrm{Ilb} / \mathrm{llla}$ and P-selectin was significantly inhibited in vitro by diltiazem in the concentration of $200 \mathrm{ng} / \mathrm{ml}$ or higher, but not 50 $\mathrm{ng} / \mathrm{ml}$.

Conclusions: Combined diltiazem and aspirin/ticlopidine therapy significantly inhibited platelet activation that continued in the presence of conventional aspirin/ticlopidine treatment. Antiplatelet effects of diltiazem were probably a consequence of reduction of platelet $\left[\mathrm{Ca}^{2+}\right.$; and may only be achieved in higher than therapeutic concentrations.
\end{abstract}

$\mathrm{P}$ athological coronary arterial thrombosis is an important complication of percutaneous transluminal coronary angioplasty and intracoronary stent implantation, which contributes to acute ischaemic events and chronic vessel restenosis. ${ }^{2}{ }^{2}$ Platelet activation in response to multiple factors by several mechanisms primarily mediates the occurrence of arterial thrombosis. ${ }^{3}{ }^{4}$ Changes in cytosolic calcium concentration play a central part in platelet activation as an upstream event which further interacts with intracellular targets to evoke functional and structural changes of platelets. ${ }^{5}$ Agents that block calcium signalling may exert their beneficial action by inhibition of platelet activation. Antiplatelet effects of calcium antagonists have been documented in vitro and in clinical studies. ${ }^{6}$ The available data indicate that diltiazem may be more potent than verapamil and the dihydropyridines in inhibiting ADP induced and collagen induced aggregation in vitro. $^{7}$ Mehta et al found that therapeutic concentrations of diltiazem attenuated thromboxane $\mathrm{B}_{2}$ generation stimulated by various agonists in platelet-rich plasma. ${ }^{8}$ Zucker et al reported that diltiazem (120 360 mg daily for a week) significantly inhibited platelet aggregation and ATP release in vitro by agonists in 25 healthy subjects. ${ }^{9}$ However, few controlled clinical in vivo studies have been performed in patients with coronary artery disease, particularly those undergoing coronary angioplasty.

Direct assessment of surface expression of integrins such as glycoprotein (GP) IIb/IIIa and P-selectin on individual platelets can be measured by whole blood flow cytometry. We investigated the expression of these antigens and changes of cytosolic calcium concentration in patients treated with diltiazem during percutaneous transluminal coronary angio- plasty (PTCA), in comparison with a control group. The purpose of the study was to evaluate effects of diltiazem on platelet hyper-reactivity in situations associated with endothelial injury and their possible relationship to cytosolic calcium concentration.

\section{SUBJECTS AND METHODS Reagents}

Diltiazem hydrochloride (Herbesser, Tanabe Seiyaku, Japan) is a benzothiazepin derivative calcium channel blocker that can be administered orally or intravenously. The following murine monoclonal antibodies were used in flow cytometry: fluorescein isothiocyanate (FITC) labelled PAC-1 (Becton Dickinson, San Jose, CA, USA), specific for activation dependent epitope of platelet GP IIb/IIIa complex; FITC labelled anti-CD62P (Fluka, Buchs, Switzerland) specific to the internal $\alpha$-granule membrane protein P-selectin expressed on the surface of activated platelets; matched controls for isotype, fluorochrome, and protein concentration were run in parallel to all monoclonal antibodies: FITC labelled IgGl (Sigma Chemical, St Louis, MO, USA) and FITC labelled IgM (Becton Dickinson, San Jose, CA, USA). The cell permeant fluorescent probe fura2/AM (fura-2 acetoxymethyl ester) for detection of cytosolic calcium concentration was from Sigma Chemical, St Louis,

Abbreviations: FITC, fluorescein isothiocyanate; GP, glycoprotein; PTCA, percutaneous transluminal coronary angioplasty 
MO, USA. Plasma thromboxane $\mathrm{B}_{2}$ was measured by radioimmunoassay using a commercial kit (RIA Institute, Beijing, China).

\section{Study groups}

Thirty five patients undergoing PTCA were studied. They were divided sequentially into a control group of 20 patients receiving aspirin/ticlopidine therapy, and a diltiazem treated group of 15 patients receiving combined diltiazem and aspirin/ ticlopidine therapy. Standard diltiazem therapy includes oral administration of 90 180 mg daily for 3 5 days before PTCA, a $10 \mathrm{mg}$ bolus injection immediately before PTCA, which is followed by a 1.5 hour, $20 \mathrm{mg} /$ hour infusion, and oral administration of 90 180 mg daily for seven days after PTCA. Both groups received standard aspirin/ticlopidine therapy which begins with ticlopidine, a $250 \mathrm{mg}$ initial dose, plus aspirin, a $300 \mathrm{mg}$ initial dose 12 to 24 hours before PTCA, which is followed by ticlopidine, $250 \mathrm{mg}$ twice daily, plus aspirin, $300 \mathrm{mg}$ daily for 30 days.

The study was approved by the ethical committee of the First Affiliated Hospital of Zhejiang University. All patients gave written consent to participate in the study.

\section{Blood sampling}

A heparin coated 5Fr Amplatz catheter was used for cannulation of the coronary sinus from the femoral vein. The two syringe method was used to obtain blood samples from the coronary sinus. Blood samples were collected at seven time points. Collection points from before PTCA included a baseline sample $<1$ hour before PTCA. At this point samples were collected from both the coronary sinus and peripheral vein. During PTCA blood samples were collected from the coronary sinus five minutes and 10 minutes after first inflation. Three other samples were obtained by venipuncture at 10 minutes, 24 hours, and 48 hours after PTCA. A preliminary study demonstrated that aspirating blood through the long catheter did not cause artificial platelet activation.

\section{Analysis of GP IIb/IIla and P-selectin expression}

Blood samples were prepared for flow cytometric analysis using the whole blood method described previously ${ }^{10}$ Five microlitres of citrated blood were added within 15 minutes after sampling to $50 \mu \mathrm{l}$ of HEPES buffered saline containing $5 \mu \mathrm{l}$ of appropriate concentrations of monoclonal antibodies. After gentle mixing, the samples were incubated for 20 minutes at room temperature, then fixed with $1 \mathrm{ml}$ of $1 \%$ paraformaldehyde to inhibit further activation.

Analysis was performed using a Coulter Epics XL flow cytometer (Coulter Electronic Ltd, Luton, UK). The platelet population was identified from its light scattering property and its identity confirmed using an isotype control.

Results are expressed as a binding index calculated from the percentage of platelets positive for the marker and the mean fluorescence intensity for each sample from the following equation: binding index $=$ (percent positive $\times$ mean fluorescence intensity) $\times 100^{-1}$.

\section{Measurement of plasma thromboxane $B_{2}$}

Thromboxane $\mathrm{B}_{2}$ was measured as the stable metabolic product of thromboxane $\mathrm{A}_{2}$ by radioimmunoassay. The technique has been described previously. ${ }^{11}$ After centrifugation at $1500 \mathrm{~g}$ for 10 minutes, supernatants were stored at $-20^{\circ} \mathrm{C}$ until analysed. A standard curve was obtained with thromboxane $\mathrm{B}_{2}$ standards. Thromboxane $\mathrm{B}_{2}$ levels in the test samples were derived from this standard curve. With this technique, the specificity is $100 \%$ for thromboxane $\mathrm{B}_{2}$. Intra-assay and interassay variation coefficients were respectively $8.7 \%$ and $10.4 \%$. All measurements were made within one month after collection of samples.

\section{Measurement of cytosolic calcium concentration in platelets}

Fresh blood was immediately mixed with $1 / 9$ by volume of citrate-citric acid-dextrose anticoagulant. Platelet-rich plasma was obtained by centrifuging whole blood at room temperature for 20 minutes at $100 \mathrm{~g}$. The platelets were sedimented by centrifugation of the supernatant at $1000 \mathrm{~g}$ for 10 minutes. The pellets were resuspended in $\mathrm{pH} 7.5$ HEPES buffered saline. The resuspended pellets were incubated with fura$2 / \mathrm{AM}\left(2 \mu \mathrm{mol} / \mathrm{l}\right.$ final concentration) for 45 minutes at $37^{\circ} \mathrm{C}$. Before the last centrifugation, apyrase was added to $20 \mu \mathrm{g} / \mathrm{ml}$. The platelets were then centrifuged at $350 \mathrm{~g}$ for 15 minutes, then resuspended in HEPES buffered saline and adjusted to a platelet count of $200 \times 10^{9} / 1$.

To measure the fluorescence, we used a Hitachi F-4000 spectrofluorometer (Hitachi Corp, Tokyo, Japan) according to the method of Grynkiewicz et al. ${ }^{12}$ The excitation wavelength was $340 \mathrm{~nm}$ and emission wavelength $500 \mathrm{~nm}$. In this study we determined the basal values for ionised calcium by using the dissociation constant and formula described by Grynkiewicz et al.

\section{In vitro studies}

Peripheral venous blood samples were collected from 10 patients with chronic stable angina (age 48-65 years) without use of a tourniquet. Patients affected by diabetes, atrial fibrillation, or haematological disease were excluded. None of the subjects had taken any platelet-active drugs in the previous 10 days. Whole blood $(0.50 \mathrm{ml})$ was incubated with normal saline (diluent for diltiazem) or with diltiazem $(50,200,500,1000$, $5000,10000 \mathrm{ng} / \mathrm{ml}$ final concentration) at $37^{\circ} \mathrm{C}$ for 1 minute. GP IIb/IIIa and P-selectin expression were analysed by whole blood flow cytometry described before.

\section{Statistical methods}

All data are shown as mean (SEM). The effects of coronary interventional procedures on the variables were compared by the paired $t$ test or non-parametric (Wilcoxon) tests. One way analysis of variance was used to determine the significance of the differences between the two groups. A p value $<0.05$ was considered significant. Data were analysed on SPSS 10.0 for Windows software.

\section{RESULTS}

\section{Clinical characteristics}

Platelet counts were normal in all patients and were not significantly affected by coronary interventional procedures (data not shown). There were no differences in baseline characteristics and procedural characteristics between control patients and those receiving diltiazem therapy (table 1). In the diltiazem treated group, intravenous diltiazem produced a reduction in mean arterial pressure (from 110.6 (1.8) to 103.2 (1.5) $\mathrm{mm} \mathrm{Hg}$ ) that was associated with a decrease in heart rate (from 86.2 (1.6) to 80.6 (1.6) beats/min).

\section{Platelet expression of GP IIb/IIla and P-selectin}

In blood samples from the coronary sinus, relative to the diltiazem treated group, platelet expression of GP IIb/IIIa significantly increased in the control group after first inflation, with a peak at 10 minutes after the inflation (fig 1A). Expression of P-selectin significantly increased relative to baseline in the control group five minutes and 10 minutes after the first inflation (fig 2A). In peripheral samples, expression of GP IIb/ IIIa and P-selectin in the control group significantly increased 10 minutes after PTCA (fig $1 \mathrm{~B}$ and 2B). These increases were not observed in diltiazem treated patients. Additionally, in the control group, at 24 hours and 48 hours after PTCA, expression of GP IIb/IIIa and P-selectin significantly decreased relative to 10 minutes after PTCA. However, in the diltiazem treated 


\begin{tabular}{|c|c|c|}
\hline Characteristic & $\begin{array}{l}\text { Control } \\
\text { patients } \\
(n=20)\end{array}$ & $\begin{array}{l}\text { Diltiazem } \\
\text { treated patients } \\
(n=15)\end{array}$ \\
\hline Mean (SEM) age in years & $63.4(2.3)$ & $67.7(1.5)$ \\
\hline Males (\%) & $16(80)$ & $13(87)$ \\
\hline Unstable angina (\%) & $16(80)$ & $12(80)$ \\
\hline Previous myocardial infarction (\%) & $4(20)$ & $1(6.7)$ \\
\hline \multicolumn{3}{|l|}{ Risk factors (\%) } \\
\hline Hypertension & $10(50)$ & $6(40)$ \\
\hline Diabetes & $3(15)$ & 0 \\
\hline \multicolumn{3}{|c|}{ Procedural variables } \\
\hline Number with three vessel disease (\%) & $5(25)$ & $3(20)$ \\
\hline Stenosis of culprit vessel in \% (SEM) & $84.4(2.1)$ & $85.7(2.4)$ \\
\hline Length of stenosis lesion in $\mathrm{mm}$ (SEM) & $15.6(1.2)$ & $18.9(1.5)$ \\
\hline Number of stents (SEM) & $1.3(0.1)$ & $1.1(0.1)$ \\
\hline $\begin{array}{l}\text { Maximal pressure of inflation (atm) } \\
\text { (SEM) }\end{array}$ & $11.8(0.7)$ & $11.2(0.4)$ \\
\hline Duration of inflation in sec (SEM) & $20.3(1.6)$ & $25.8(3.0)$ \\
\hline Dose of heparin in $\mathrm{mg}$ (SEM) & $73.6(3.9)$ & $64.6(3.3)$ \\
\hline
\end{tabular}
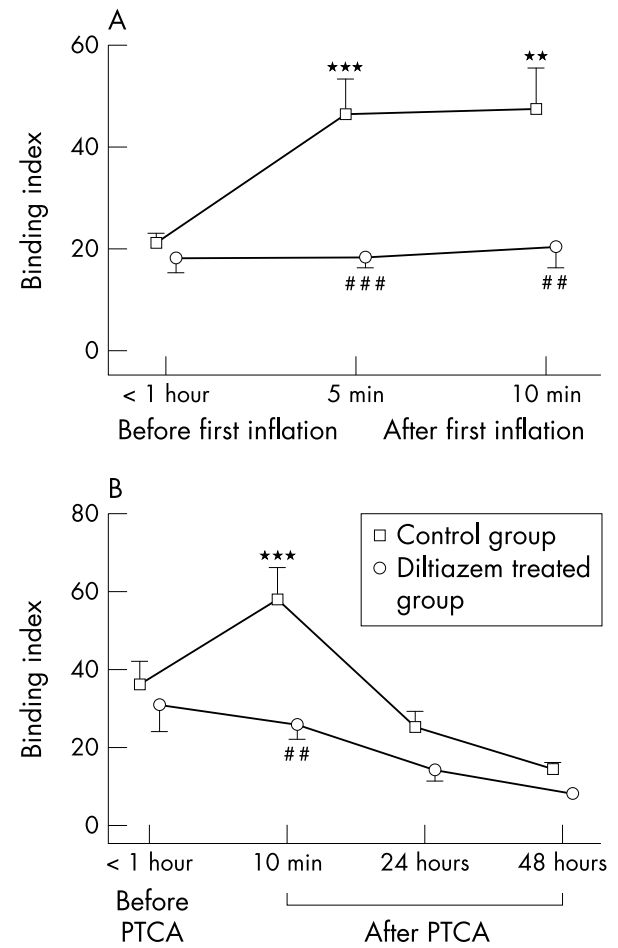

Figure 1 Platelet expression of GP Illb/llla in control group or diltiazem treated group during PTCA from coronary sinus samples (A) or peripheral samples (B). Values are mean (SEM). Difference between means is significant between baseline and time point $\left({ }^{*} p<0.01,{ }^{* *} p<0.001\right)$, and diltiazem treated group and control group (\# \#p<0.01, \# \# \#p<0.001).

group, at 24 hours and 48 hours after PTCA, expression of GP IIb/IIIa and P-selectin significantly decreased relative to baseline (fig $1 \mathrm{~B}$ and $2 \mathrm{~B}$ ).

\section{Plasma thromboxane $B_{2}$}

Plasma thromboxane $B_{2}$ significantly increased 10 minutes after the first inflation in the control group. In diltiazem treated patients, plasma thromboxane $\mathrm{B}_{2}$ was significantly less than that in control patients five minutes and 10 minutes after first inflation (fig 3A). In the control group, plasma thromboxane $\mathrm{B}_{2}$ significantly increased 10 minutes after PTCA. This increase was not observed in diltiazem treated patients. In the control group, at 48 hours after PTCA, plasma
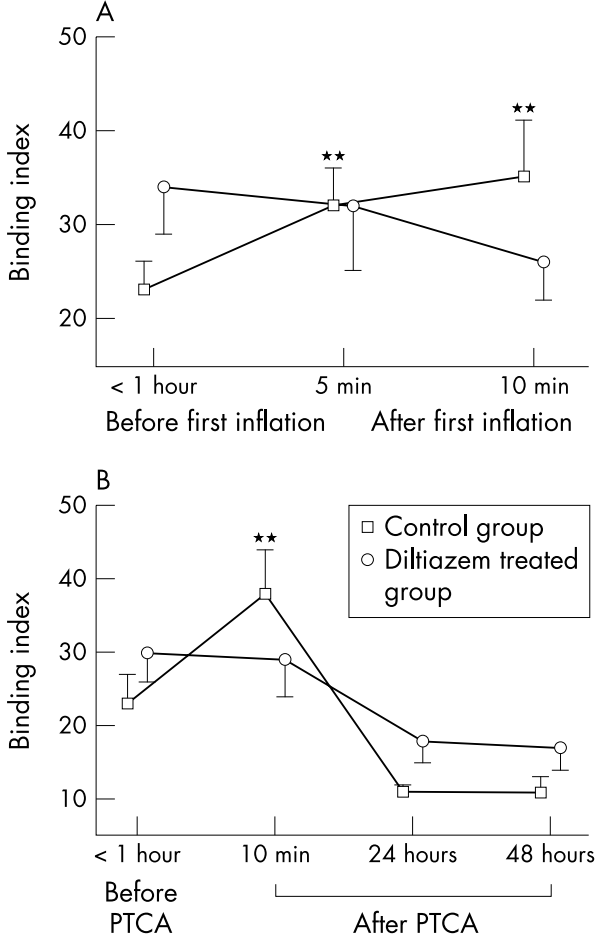

Figure 2 Platelet expression of $\mathrm{P}$-selectin in control group or diltiazem treated group during PTCA from coronary sinus samples (A) or peripheral samples (B). Values are mean (SEM). Difference between means is significant between baseline and time point $\left({ }^{* *} p<0.01\right)$.

thromboxane $\mathrm{B}_{2}$ significantly decreased relative to 10 minutes after PTCA (fig 3B).

\section{Cytosolic calcium concentration}

Platelet $\left[\mathrm{Ca}^{2+}\right]_{\mathrm{i}}$ significantly increased after first inflation in the control group, with a peak at 10 minutes after the inflation. Relative to aspirin/ticlopidine therapy alone, platelet $\left[\mathrm{Ca}^{2+}\right]_{\mathrm{i}}$ was significantly lowered with combined diltiazem and aspirin/ticlopidine therapy five minutes and 10 minutes after first inflation (fig 4A). Only in the control group, platelet $\left[\mathrm{Ca}^{2+}\right]_{\mathrm{i}}$ significantly increased 10 minutes after PTCA. In both groups, at 24 hours and 48 hours after PTCA, platelet $\left[\mathrm{Ca}^{2+}\right]_{\mathrm{i}}$ significantly decreased relative to 10 minutes after PTCA (fig $4 B)$.

\section{In vitro studies}

Expression of GP IIb/IIIa and P-selectin was not significantly inhibited by diltiazem in the concentration of $50 \mathrm{ng} / \mathrm{ml}$, but in the concentrations of $200 \mathrm{ng} / \mathrm{ml}$ or $500 \mathrm{ng} / \mathrm{ml}$ (data not shown). A representative experiment is shown in fig 5 . Higher concentrations ( $\geqslant 1 \mu \mathrm{g} / \mathrm{ml}$ ) of diltiazem almost inhibited their expression completely.

\section{DISCUSSION}

We have shown a time dependent increase of the parameters of platelet activation after PTCA; the increase of platelet expression of GP IIb/IIIa and P-selectin peaked 10 minutes after first inflation in coronary sinus samples; expression of GP IIb/IIIa and P-selectin and plasma thromboxane $\mathrm{B}_{2}$ increased 10 minutes after PTCA in peripheral samples, with a decrease at 24 hours and 48 hours.

These data demonstrate that systemic and local platelet activation occurs after coronary interventional procedures despite conventional aspirin/ticlopidine treatment. In contrast to our present findings, Fredrickson et al showed no increased platelet activation in peripheral blood samples from patients 

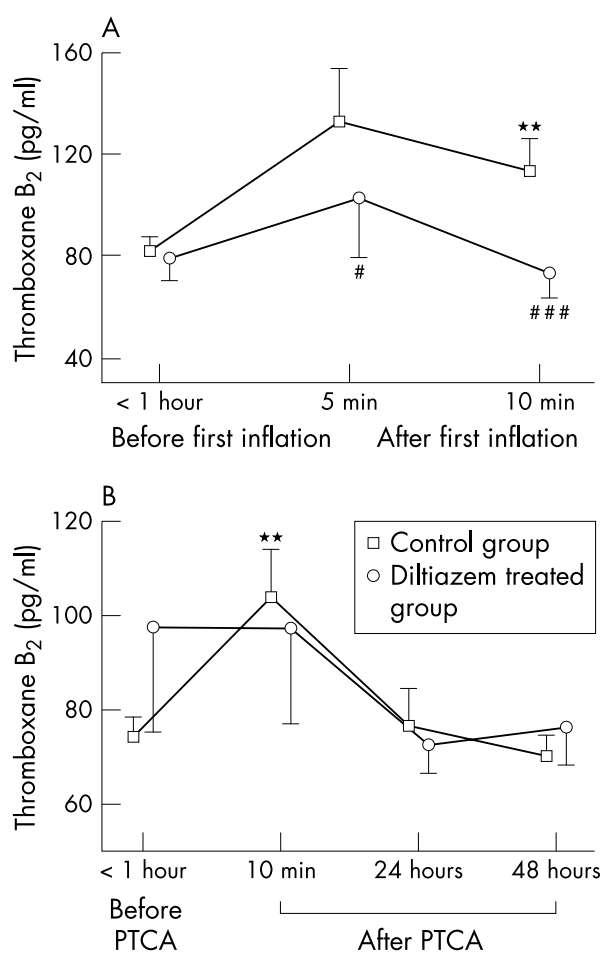

Figure 3 Plasma thromboxane $B_{2}$ in control group or diltiazem treated group during PTCA from coronary sinus samples (A) or peripheral samples (B). Values are mean (SEM). Difference between means is significant between baseline and time point $\left({ }^{*} p<0.01\right)$, and diltiazem treated group and control group $(\# p<0.05$, \# \# \#p<0.001).

undergoing elective PTCA, but the number of subjects in the study was too small and samplings were performed much later, at a time when acute changes might have been attenuated by the rapid clearance of activated platelets from the circulation. ${ }^{13}$ Differences in outcomes of studies may also be due to our standardised protocol, local sampling techniques, and frequent sampling.

Although there is no any evidence of a direct effect of ticlopidine on the GP IIb/IIIa complex, previous studies reported that the addition of ticlopidine for $\geqslant 3$ days to aspirin effectively inhibited expression of GP Iib/IIIa. ${ }^{14}$ Our results would therefore agree with most of these studies, in that administration of initial dose of ticlopidine 12 24 hours before PTCA failed to prevent an acute rise of the parameters of platelet activation as a consequence of the vascular injury after elective PTCA. ${ }^{16}$ Since continuing platelet activation contributes to an adverse outcome, this suggests that additional antiplatelet therapy is necessary. However, all parameters fell to close to or below baseline values 24 hours and 48 hours after PTCA, suggesting the slow effect of ticlopidine on platelet activation and systemic blood dilution to the local plaque event.

Elevation of intracellular calcium concentration, either as a result of calcium influx from the exterior or calcium release from intracellular stores, is a necessary and sufficient event for platelet activation. ${ }^{17}$ Previous studies showed that calcium antagonists have an inhibitory effect on platelet activation in vitro and ex vivo, but no clear consensus on concentrations that the inhibition requires has been reached from existing studies. We showed that, after incubation with higher than therapeutic concentrations of diltiazem $(200 \sim 500 \mathrm{ng} / \mathrm{ml})$ in vitro, expression of GP IIb/IIIa and P-selectin was significantly inhibited in whole blood samples from patients with chronic stable angina. The inhibition of platelet activation with diltiazem occurs in a concentration dependent fashion. Although diltiazem in therapeutic concentrations may exert
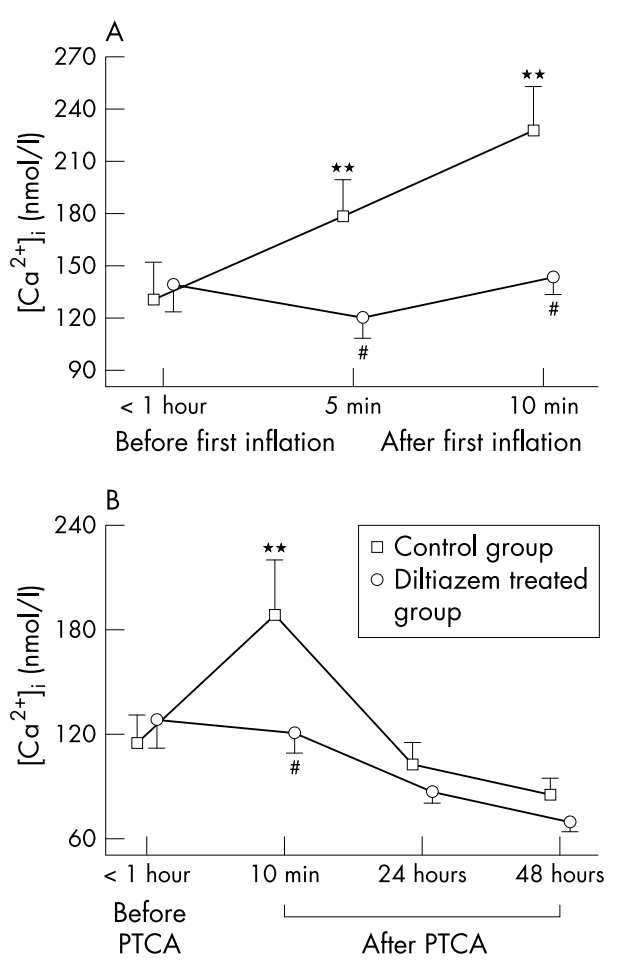

Figure 4 Platelet $\left[\mathrm{Ca}^{2+}\right]_{i}$ in control group or diltiazem treated group during PTCA from coronary sinus samples (A) or peripheral samples (B). Values are mean (SEM). Difference between means is significant between baseline and time point $\left({ }^{*} p<0.01\right)$, and diltiazem treated group and control group $(\# p<0.05)$.

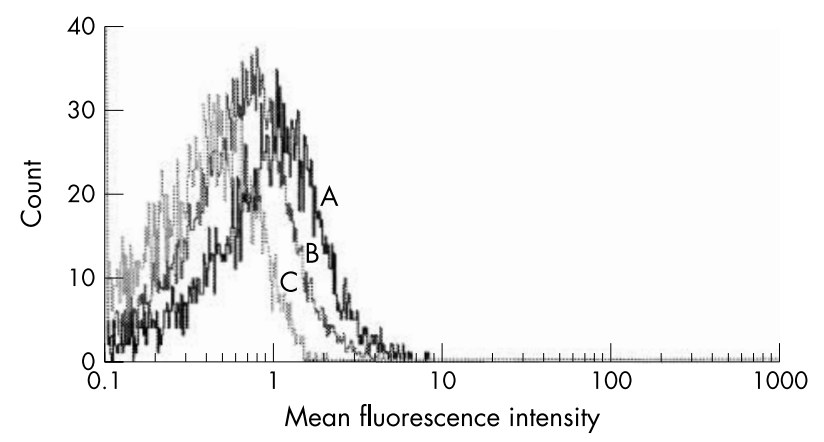

Figure 5 Effect of diltiazem on platelet expression of GP Ilb/Illa in vitro in a representative whole blood sample from a patient with chronic stable angina. A, no diltiazem; $B, 200 \mathrm{ng} / \mathrm{ml}$ diltiazem 10.4 $\mu M) ; C, 1 \mu g / m l$ diltiazem (2.2 $\mu M)$.

modest inhibition on thromboxane $\mathrm{B}_{2}$ generation and secondary aggregation induced by stimuli in subthreshold concentrations, our present study suggested that inhibition of fibrinogen binding and degranulation of $\alpha$-granules can only be achieved in higher concentrations.

We added diltiazem by oral administration and infusion during PTCA and found that platelet activation occurring in the presence of standard antiplatelet treatment was prevented by this calcium antagonist. However, Knight et al rerported that in healthy volunteers there was a rise in P-selectin expression and GP IIb/IIIa levels were not affected after oral administration of amlodipine for one week. ${ }^{18}$ The inconsistencies were not surprising and have several possible explanations. First, using a combination of oral administration and infusion, we obtained high blood drug concentrations of diltiazem during the interventional procedure that may exert a measurable effect on platelet function in vivo. But the regimen has been shown to cause a mild reduction of heart rate and mean arterial pressure with the starting infusion dose. 
Second, patients with coronary artery disease, particularly those undergoing coronary interventional procedures, have abnormal platelet responsiveness resulting from functionally incompetent endothelium and unstable plaques. ${ }^{19}$ Their response to calcium antagonists may therefore differ from that observed in healthy subjects. For example, Sanguigni et al reported inhibition of exercise induced increase of expression of P-selectin after amlodipine in patients with chronic stable angina. ${ }^{20}$

Our study, using the sensitive fluorescent probe for the first time to investigate changes in cytosolic calcium concentration of platelets during PTCA, found that platelet $\left[\mathrm{Ca}^{2+}\right]_{\mathrm{i}}$ significantly increased immediately after PTCA, with an obvious decrease 24 hours after PTCA. There was a statistical correlation between the changes in platelet $\left[\mathrm{Ca}^{2+}\right]_{\mathrm{i}}$ and those in the parameters of platelet activation measured. This substantiated the speculation that changes in $\left[\mathrm{Ca}^{2+}\right]_{\mathrm{i}}$ are fundamental to the platelet response to activation in clinical complicated settings as seen in vitro. We also observed that an angioplasty induced short term increase of platelet $\left[\mathrm{Ca}^{2+}\right]_{i}$ was significantly reduced by diltiazem. In vitro studies, however, showed that diltiazem, verapamil, and nifedipine inhibited $\mathrm{Ca}^{2+}$ influx induced by ADP, platelet activating factor, and U46619 in concentrations far above those achieved with usual clinical use. ${ }^{21}$ Our results may relate to high concentrations of diltiazem obtained and relatively high platelet activation status in patients with coronary arterial disease. Unlike excitable cells, human platelets do not possess voltage dependent calcium channels and there is still lack of consensus on clear mechanisms of calcium influx..$^{22}{ }^{23}$ It has been proposed that calcium antagonists may exert their effects on platelet $\left[\mathrm{Ca}^{2+}\right]$ by inhibition of calcium mobilization from intracellular stores, inhibition of GP IIb/IIIa complex, or interaction with receptor operated calcium channels such as $\alpha$-adrenergic receptors. ${ }^{24}$

Platelet activation after PTCA is generally associated with an adverse prognosis and is one of the primary targets of medication. We demonstrated that platelet activation that continued in the presence of conventional aspirin/ticlopidine treatment was inhibited by combined diltiazem and aspirin/ ticlopidine treatment. Although accurate mechanisms of calcium influx are still unclear, inhibition of an increase of platelet $\left[\mathrm{Ca}^{2+}\right]_{i}$ by calcium antagonists may represent a striking approach to the prevention of platelet activation after coronary interventional procedures. Future studies should include platelet specific and efficient calcium antagonists to evaluate their use.

\section{ACKNOWLEDGEMENTS}

This work was supported by a grant from Health Bureau of Zhejiang province. We are grateful to Minwei Li and Wentao Jing for technical assistance.

\section{Authors' affiliations}

H Dai, J Chen, Q Tao, J Zhu, F Zhang, L Zheng, Y Qiv, Department of Cardiology, First Affiliated Hospital of Zhejiang University, Hangzhou, Zhejiang, China

\section{REFERENCES}

1 Landau C, Lange RA, Hillis DH. Percutaneous transluminal coronary angioplasty. N Engl J Med 1994;330:981-93.

2 Oesterle SN, Whitbourn R, Fitzgerald PJ, et al. The stent decade: 1987 to 1997. Am Heart J 1998;136:578-99.

3 Gasperetti CM, Gonias SL, Gimple LW, et al. Platelet activation during coronary angioplasty in humans. Circulation 1993;88:2728-34.

4 Vaitkus PT, Watkins MW, Witmer WT, et al. Characterization of platelet activation and thrombin generation accompanying percutaneous transluminal coronary angioplasty. Coron Artery Dis 1995;6:587-92.

5 Rink TJ. Cytosolic calcium in platelet activation. Experientia 1988:44:97-100

6 Hiemdahl $\mathbf{P}$, Wallen NH. Calcium antagonist treatment, sympathetic activity and platelet function. Eur Heart J 1997;18(suppl A):A36-50.

7 Walley TJ, Woods KL, Barnett DB. Effects of calcium channels blockers on in vitro platelet function in whole blood using single platelet counting. Thromb Haemost 1989;61:137-9.

8 Mehta J, Mehta P, Ostrowski N. Calcium blocker diltiazem inhibits platelet activation and stimulates vascular prostacyclin synthesis. Am J Med Sci 1986:291:20-4.

9 Zucker ML, Budd SE, Dollar LE, et al. Effects of diltiazem and low-dose aspirin on platelet aggregation and ATP release induced by paired agonists. Thromb Haemost 1993:70:332-5.

10 Shattil SJ, Cunningham M, Hoxie JA. Detection of activated platelets in whole blood using activation dependent monoclonal antibodies and flow cytometry. Blood 1987;70:307-15

$11 \mathrm{Koh} H$, Inove $A$, Mashimo N, et al. A radioimmunoassay of thromboxane $\mathrm{B}_{2}$ with thromboxane $\mathrm{B}_{2}{ }^{-125}$-tyramide and its application to the study on the thromboxane $B_{2}$ formation during platelet aggregation. Thromb Res 1980;17:403-13.

12 Grynkiewicz G, Poenie M, Tsien RY. A new generation of $\mathrm{Ca}^{2+}$ indicators with greatly improved fluorescence properties. J Biol Chem 1985;260:3440-50.

13 Fredrickson BJ, Turner NA, Kleiman NS, et al. Effects of Abciximab, ticlopidine, and combined Abciximab/ticlopidine therapy on platelet and leukocyte function in patients undergoing coronary angioplasty. Circulation 2000:101:1122-9.

14 Neumann FJ, Gawaz M, Dickfeld T, et al. Antiplatelet effect of ticlopidine after coronary stenting. J Am Coll Cardiol 1997;29:1515-9.

15 Gregorini L, Marco J, Fajadet J, et al. Ticlopidine and aspirin pretreatment reduces coagulation and platelet activation during coronary dilation procedures. J Am Coll Cardiol 1997;29:13-20.

16 Mizuno O, Hojo Y, Ikeda U, et al. Assessment of coagulation and platelet activation in coronary sinus blood induced by transcatheter coronary intervention for narrowing of the left anterior descending coronary artery. Am J Cardiol 2000;85: 154-60.

17 Rink TJ, Sage SO. Calcium signaling in human platelets. Annu Rev Physiol 1990;52:431-49.

18 Knight CJ, Panesar M, Wilson DJ, et al. Different effects of calcium antagonists, nitrates, and $\beta$-blockers on platelet function. Possible importance for the treatment of unstable angina. Circulation 1997; 95: 125-32.

19 Katopodis JN, Kolodny L, Jy W, et al. Platelets microparticles and calcium homeostasis in acute coronary ischemias. Am J Hematol 1997:54:95-101.

20 Sanguigni V, Gallu M, Sciarra L, et al. Effect of amlodipine on exercise-induced platelet activation in patients affected by chronic stable angina. Clin Cardiol 1999;22:575-80.

21 Rostagno C, Abbate R, Gensini GF, et al. In vitro effects of two novel calcium antagonists (nitrendipine and nisoldipine) on intraplatelet calcium redistribution, platelet aggregation and thromboxane $\mathrm{A}_{2}$ formation. Comparison with diltiazem, nifedipine and verapamil. Thromb Res 1991;63:457-62.

22 Pannocchia A, Praloran N, Arduino C, et al. Absence of $\left[{ }^{3} \mathrm{H}\right]$ desmethoxy-verapamil binding sites on human platelets and lack of evidence for voltage-dependent calcium channels. Eur J Pharmacol 1987:142:83-91.

23 Putney JW, Jr Broad LM, Braun F-J, et al. Mechanisms of capacitative calcium entry. J Cell Sci 2001;114:2223-9.

24 Rybak MEM, Renzulli LA. Effect of calcium channel blockers on platelet GP Ilb/llla as a calcium channel in liposomes: comparison with effects on the intact platelet. Thromb Haemost 1992;67:131-6. 